\title{
"Cataplexy" and muscle ultrasound abnormalities in Coffin-Lowry syndrome
}

\author{
Y J Crow, S M Zuberi, R McWilliam, J L Tolmie, A Hollman, K Pohl, J B P Stephenson
}

\begin{abstract}
The Coffin-Lowry syndrome is a rare cause of mental retardation recognised by its distinctive facial and digital features. We have observed an unusual, nonepileptic, cataplexy-like phenomenon in three subjects with the syndrome and we speculate that this feature may go unrecognised. We also provide evidence of neuromuscular dysfunction as part of the phenotype by showing abnormalities on muscle ultrasound in four gene carriers. $(\mathcal{M}$ Med Genet 1998;35:94-98)
\end{abstract}

Keywords: Coffin-Lowry syndrome; cataplexy; muscle ultrasound abnormalities

Coffin $e t a l^{1}$ in 1966 and Lowry et al in 1971 independently described patients with the mental handicap syndrome which now bears their names. They recognised a phenotype comprising moderate to severe mental retardation, facial dysmorphism, tapering digits, and skeletal deformity. ${ }^{3}$ Linkage analysis subse-

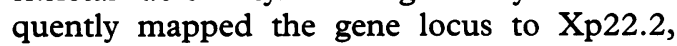
supporting the clinical impression that the syndrome is an $\mathrm{X}$ linked trait with variable clinical manifestations in female heterozygotes. Recently, mutations have been identified in the Rsk-2 gene in affected subjects. ${ }^{4}$ Here we report five patients with Coffin-Lowry syndrome from four families. Three of them exhibit episodes of sudden, non-epileptic collapse with atonia which we describe as a form of cataplexy. Four patients show significant changes on muscle ultrasound indicative of undefined neuromuscular dysfunction. Neither of these features has been previously recognised in this syndrome.

\section{Case reports}

CASE 1 (MALE)

Scoliosis was evident by the age of 6 months and significant developmental delay was obvious by 9 months of age. When 6 years old, he developed episodes of sudden loss of tone causing his legs to give way under him resulting in recurrent falls to the ground. These episodes were reported to be precipitated by a loud noise and could also occur at times of excitement and after being "told off". Tone was regained within a few seconds. There were no associated jerking movements. The frequency of the attacks subsequently necessitated the use of a wheelchair from the age of 10 years. At 14 years of age he was referred for detailed neurological assessment. It was felt that his facial features and fingers were in keeping with a diagnosis of Coffin-Lowry syndrome (figs 1 and 2). Investi- gation of urinary glycosaminoglycans, chromosomes, and DNA for fragile $\mathrm{X}$ syndrome was unremarkable. Close examination of video recordings of the episodes suggested a loss of tone spreading from the head downwards with an initial drooping of his eyelids, followed by sagging of the jaw, dropping of the head towards the chest, and finally a falling forward of his body so that he appeared to "slump" into his chair. Although muscle power appeared normal, muscle ultrasound (recorded using an Acuson 128XP 10 ultrasound system with 7 $\mathrm{MHz}$ probe) showed increased muscle echogenicity, reduced bone echogenicity, a reduction in muscle bulk, and an increase in subcutaneous fat (fig 3). These changes were classified as grade 3 according to the system suggested by Heckmatt et al. ${ }^{5}$ Serum creatine kinase was normal. Motor and sensory nerve conduction velocities measured in the right median nerve were normal. Limited needle EMG examination of the right first dorsal interosseous muscle showed normal wave form morphology although of lower than expected amplitude ( $<1$ mvolt). A full interference pattern was obtained but again the amplitude was lower than expected at 2.5 mvolts. There was no spontaneous activity or increased insertional activity. Needle muscle biopsy of the right vastus lateralis, including electron microscopy and dystrophin immunocytochemistry, was unremarkable. EEG during one of these

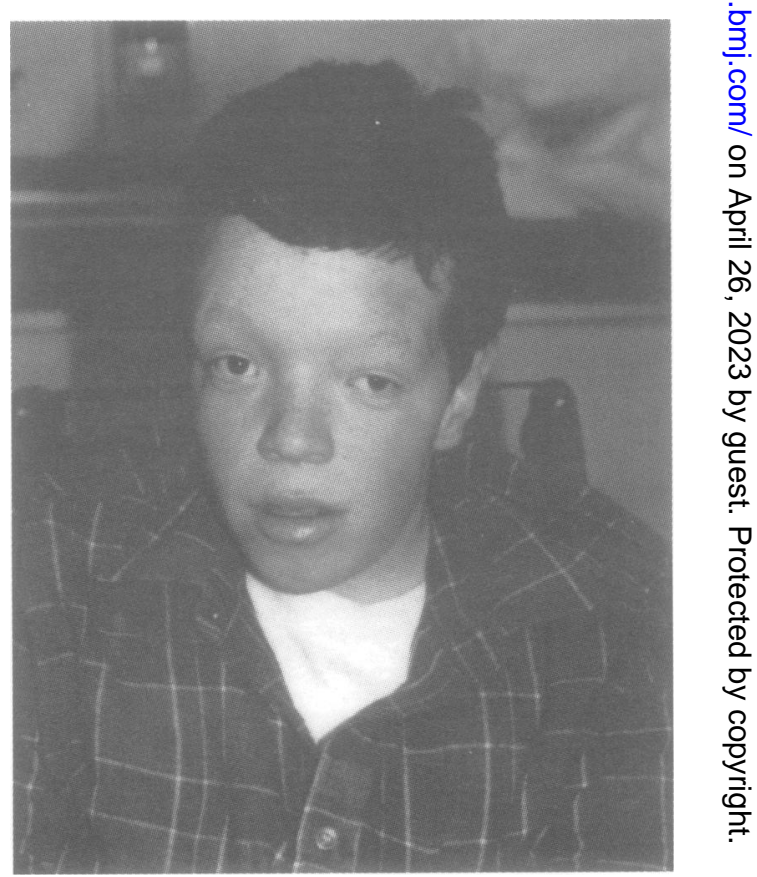

Figure 1 Case 1 showing typical facial features of Coffin-Lowry syndrome. (Photograph reproduced with permission.) 


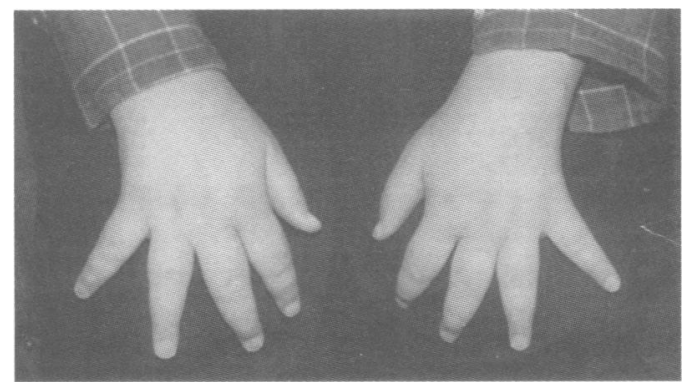

Figure 2 Case 1 showing typical digital features of Coffin-Lowry syndrome.

induced falls did not show any epileptic discharges (fig 4). Changes in paraspinal muscle activity were assessed using surface electrodes placed $2 \mathrm{~cm}$ either side of the midline at C5 level with a ground electrode on the right forearm. Collapses could be induced by sudden and unexpected sound or electrical stimulation of a peripheral nerve above the motor threshold. Following successful stimulation, there was an abrupt reduction in surface EMG activity with a latency of approximately $76 \mathrm{msec}$. This reduction was transient, $4 \mathrm{msec}$, and was followed by an increase in EMG activity to greater than pre-stimulus levels.

\section{CASE 2 (MALE)}

At the age of 1 year he was noted to be dysmorphic and by 16 months was showing signs of global developmental delay. Chromosomal analysis was normal. He was diagnosed with Coffin-Lowry syndrome when 5 years old. At the age of 7 years, it was noted that a sudden noise would make him fall: "if startled his legs switch off". He would then get up and walk again as normal. An EEG was unremarkable. By the age of 10 years these episodes were occurring up to 12 times per day. When standing or walking he would suddenly drop, always in a forwards direction, and never from a sitting position. On occasion, he would "half-drop then freeze", being able to recover before collapsing completely. On other occasions his falls were so instantaneous that he hurt himself, dislocating his jaw and fracturing his
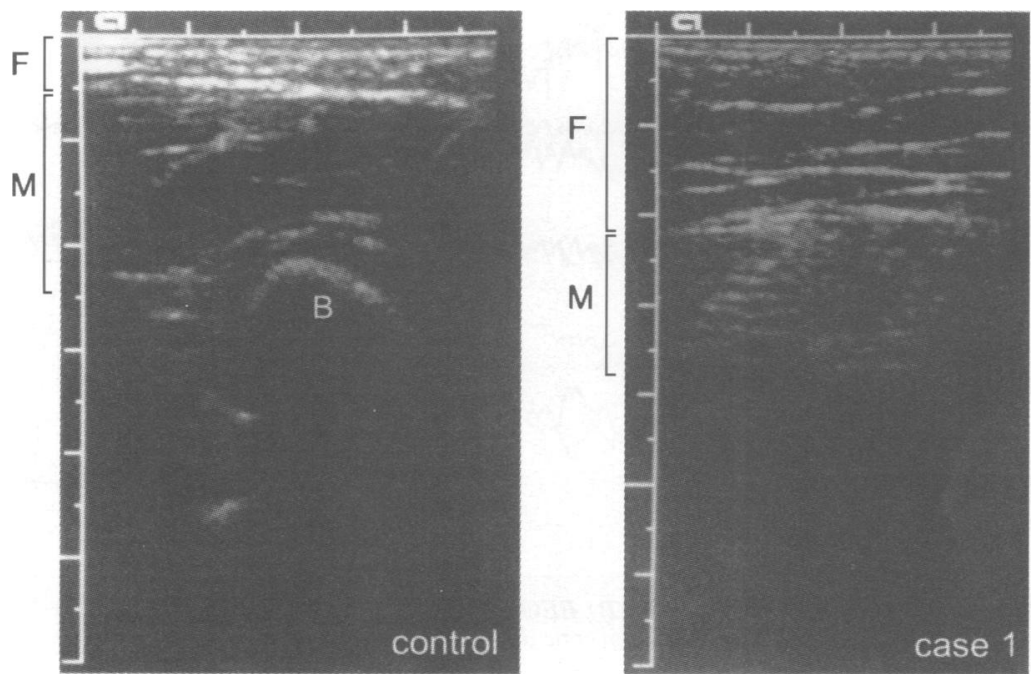

Figure 3 Muscle ultrasound of thigh. Appearance in case 1 (right) shows a reduction in bone echo (B), a reduction in muscle bulk with increased echogenicity (M), and an increase in subcutaneous fat $(F)$ compared to normal control (left). nose. He recovered spontaneously from these episodes which were not accompanied by confusion or loss of consciousness. EEG, MRI of the brain, cerebral angiography, and ECG were all normal. Muscle ultrasound was unremarkable. A therapeutic trial of clobazam did not produce any clear benefit.

\section{CASE 3 (FEMALE)}

Unfortunately, few details are available for this patient regarding family history and early development. However, it is known that she had significant developmental retardation from a young age and was placed in a psychiatric institution. Frequent attacks of falling down were recognised from the age of 13 years. When placed on her feet she was said to "buckle at the knees" and fall to the ground. Initially it was felt that her walking difficulties were non-organic in nature. At the age of 35 years she was reassessed. Facial and digital features were consistent with a diagnosis of Coffin-Lowry syndrome (fig 5). Muscle ultrasound showed generalised increase in muscle echogenicity and reduction in muscle bulk (grade 4, Heckmatt). These changes were most striking in the calves and thighs. The arms and forearms were least affected. Serum creatine kinase was normal. $X$ ray of the hands showed typical tufting of the terminal phalanges. Attempts at recording surface EMG activity during an episode of collapse were unsuccessful.

\section{CASE 4 (MALE)}

Developmental delay was first noted at the age of 11 months with an inability to sit unsupported. At 17 months of age there was no understanding of spoken language and no symbolic play. He was still unsteady when sitting and was unable to roll or stand. His OFC was $47 \mathrm{~cm}$ (10-50th centile). $\mathrm{He}$ was noted to have plagiocephaly, tapering fingers, and a dysmorphic facies consistent with Coffin-Lowry syndrome. Muscle ultrasound showed a marked increase in muscle echogenicity and a reduction in bone echogenicity (grade 3, Heckmatt) particularly affecting the thighs and calves but also, to a lesser extent, the arms and forearms. Serum creatine kinase and chromosomal analysis were both normal. At 30 months he is making some developmental progress, is now able to walk unsteadily, and shows limited understanding of symbolic play.

\section{CASE 5 (FEMALE)}

This was the mother of case 4 . She had a poor educational record associated with learning difficulties. She has tapering fingers with prominence of the terminal phalanges on $x$ ray consistent with carrier status for Coffin-Lowry syndrome. Muscle ultrasound showed generalised increase in muscle echogenicity with reduction in muscle bulk predominantly affecting the thighs and calves (grade 3 , Heckmatt). Her mother was also said to have fingers which were broad proximally but tapering towards the ends. 

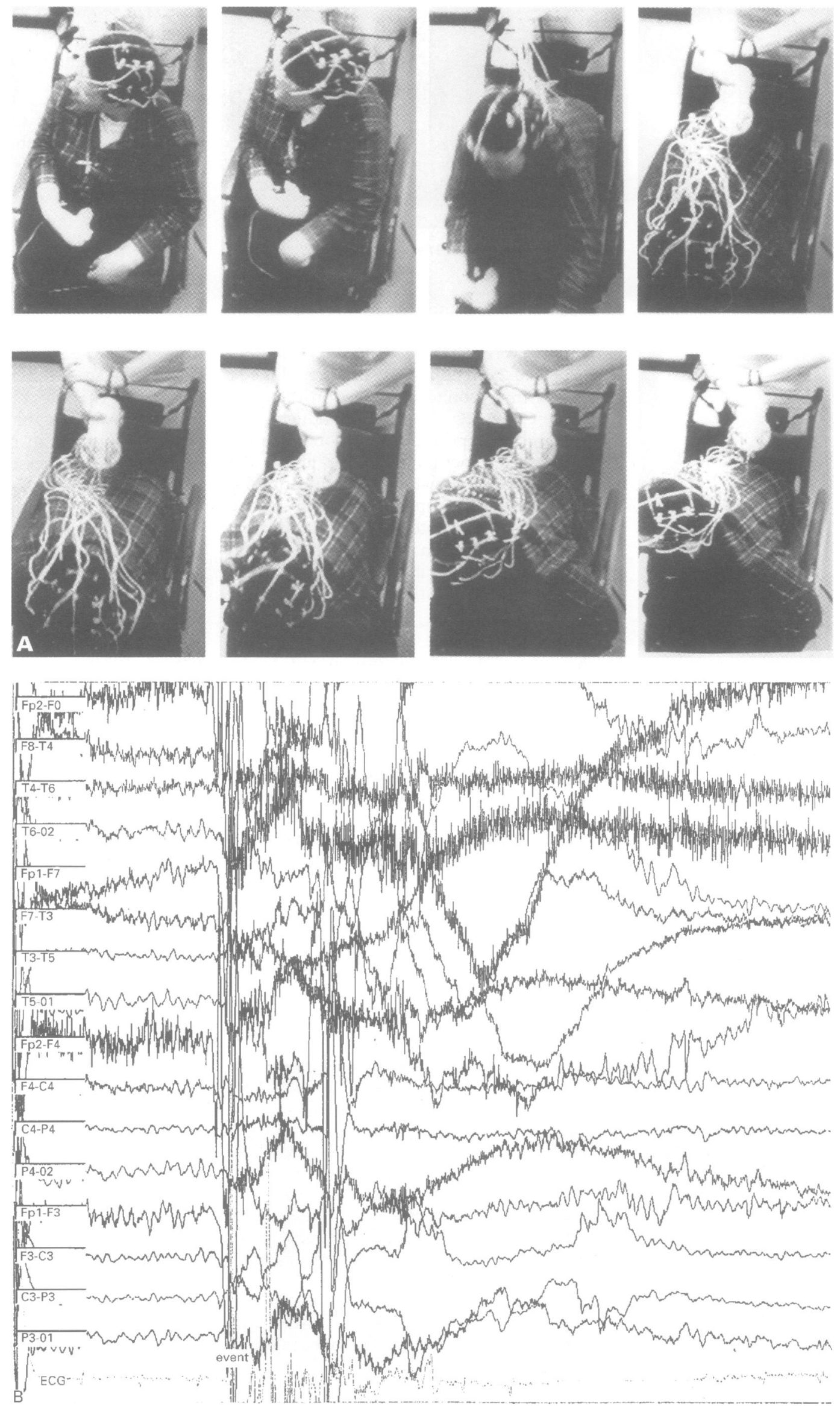

Figure 4(A) Serial frames at 400 msec intervals after sound startle. (B) EEG during sound startle induced collapse displays large motion artefact and scalp muscle potential but neither epileptic discharges nor background suppression were evident. Interictal EEG was also normal. 


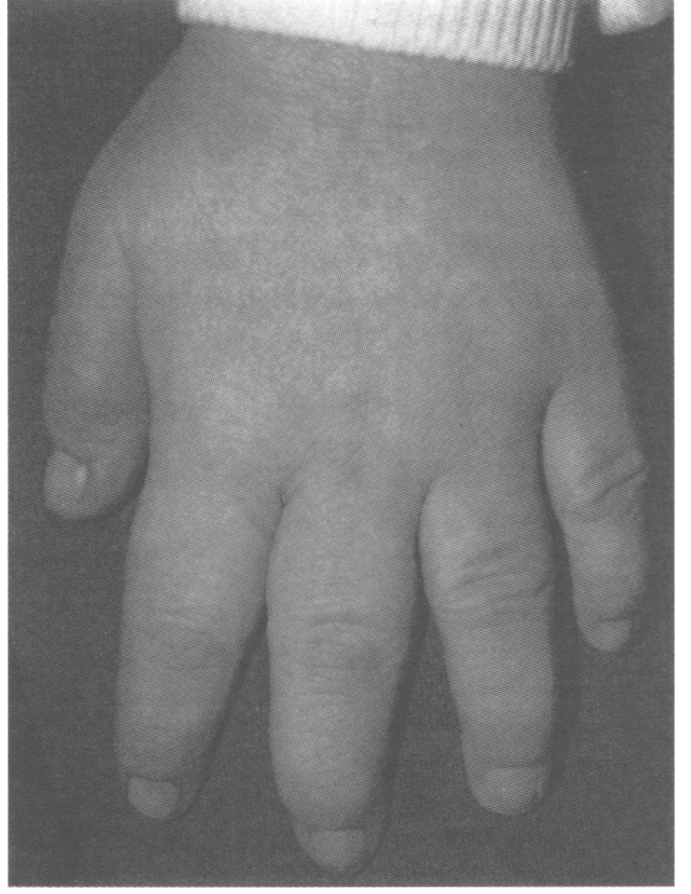

Figure 5 Typical digital changes seen in a manifesting female (case 3).

\section{Discussion}

In this report we describe three subjects with Coffin-Lowry syndrome who show an unusual phenomenon characterised by a sudden and reversible loss of muscle tone without loss of consciousness. This developed in two males beginning at 6 and 7 years of age and in one manifesting female heterozygote beginning at the age of 13 years. The frequency of attacks has resulted in all three patients being confined to a wheelchair to reduce the risk of injury, although there is no evidence to suggest that collapses become more frequent or severe with time. We could find only one other description of a person with Coffin-Lowry syndrome and recurrent collapses that may be similar to those seen in three of our patients. Ishida $e t a l^{\circ}$ describe a male (case 2) who "occasionally lost consciousness, but electrocardiogram and electroencephalogram showed normal patterns".

The major differential diagnosis of collapse in these three patients includes an epileptic phenomenon, hyperekplexia, negative myoclonus, and cataplexy.

"Seizures" have been described in patients with Coffin-Lowry syndrome, ${ }^{278}$ although descriptions of the episodes and documentation of the EEG findings are frequently inadequate. The normal EEG recorded during episodes in two of our cases suggests that they are not epileptic in origin. Although startle provoked epileptic seizures (SPES) can occur in the absence of EEG changes, the clinical features are quite distinct. In particular, in SPES, seizures are almost always also seen in the absence of startle provocation and are characterised by focal or generalised tonic motor activity. ${ }^{9}$ Neither of these features was seen in the subjects we describe.

Hyperekplexia is characterised by an exaggerated motor response to a sudden and unexpected noise, a tap to the body or, less frequently, a visual stimulus. Typically, the patients will "startle" and if standing may fall and injure themselves. This exaggeration of the normal startle reflex is accompanied by hypertonia and a marked increase of muscle activity on EMG recording, ${ }^{10}$ which features differentiate it completely from the episodes reported here.

Negative myoclonus, asterixis, is characterised by arrhythmic lapses of sustained posture with periods of EMG silence lasting up to 400 msec. It is not associated with startle provocation and is usually seen in the context of metabolic and toxic encephalopathies. ${ }^{11}$

Cataplexy comprises an abrupt, complete, or partial loss of voluntary muscle tone and is usually seen in response to strong emotion but can be precipitated by startle. It is rare in childhood and has previously only been described in association with narcolepsy, ${ }^{12}$ Niemann-Pick type $\mathrm{C},{ }^{13}$ Prader-Willi syndrome, ${ }^{14}$ and Norrie disease. ${ }^{15}$ Cataplexy in association with the narcolepsy syndrome is characterised by typical EEG correlates of REM sleep which were not seen in our cases. We also looked for the HLA DR2/DQ $6{ }^{16}$ haplotype in two patients and this was positive in case 1 only. Vossler $e t a^{15}$ described cataplexy in boys with Norrie disease and a reduction in monoamine oxidase type B (MAO-B). We measured platelet monamine oxidase activity in case 1 and the level was unremarkable. Guilleminault and $\mathrm{Gelb}^{17}$ have described the electrophysiological characteristics of cataplexy which include an abolition of the $\mathrm{H}$ reflex during attacks of atonia. In view of the very short duration of the episodes seen in our patients we were unable to replicate these studies. We were, however, able to show changes in paraspinal muscle activity which suggest that, in response to a sudden and unexpected stimulus, the initial event is a loss of muscle tone followed thereafter by a very rapid increase in muscle activity.

The short duration of the attacks we describe and their association with sound/startle provocation are not typical of cataplexy. However, the features seen on the video recording of case 1 are very similar to those seen in cataplexy and it is important to note that emotional stress is also described as a trigger in case 1 . We regard the observation in case 2 that, on occasion, he would "half drop then freeze" as typical of some episodes seen in cataplexy where there appears to be a successful voluntary attempt to overcome the atonic attack. For these reasons we feel that cataplexy is the best description of these collapses. Moreover, the occurrence of this unusual cataplexy-like phenomenon in three subjects with Coffin-Lowry syndrome suggests to us that it might be a relatively common feature of the condition and that episodes previously described as epileptic may, in fact, be similar to those we report.

Heckmatt et $a l^{18}$ in 1980 first described the use of ultrasound imaging to detect pathological changes in the muscles of boys with Duchenne muscular dystrophy. They reported increased echogenicity of damaged muscle, as a consequence of progressive infiltration and 
replacement of muscle by adipose and connective tissue, and later extended these observations to other neuromuscular conditions. Importantly, they found that controls showed clear delineation of bone and fascia, appearing as strongly echogenic structures, while muscle was only weakly echogenic.

We have observed abnormalities on muscle ultrasound in four of our patients with increase in muscle echogenicity (especially in the thighs and calves), variable attenuation of bone echo, reduction in muscle bulk, and increase in subcutaneous fat. These changes are similar to those seen in spinal muscular atrophy. Grade 3 changes were seen in a 2 year old male and grade 4 abnormalities were present in a manifesting female at the age of 35 years. It is difficult to determine the degree of functional weakness and whether or not it is progressive, but it is well recognised that the severity of the changes observed on ultrasound do not necessarily relate to the degree of functional disability. Abnormalities of muscle on ultrasound were not seen in case 2 and are presumably not related to the cataplexy-like episodes.

Muscle wasting and weakness are not commonly referred to as a part of the clinical phenotype in Coffin-Lowry syndrome. However, Fryns et al described two brothers with Coffin-Lowry syndrome who showed marked distal muscle wasting. Other authors have also commented on this aspect of the condition. ${ }^{19-21}$ Kyphoscoliosis, pes planus, and pectus deformities are well recognised and it is possible that these features may be the result, at least in part, of neuromuscular dysfunction. Investigations to elucidate the nature of any nerve or muscle disorder have included creatine kinase estimation, electromyography, muscle biopsy, and nerve conduction studies. Vine et $a l^{2}$ reported "diminished amplitude and prolonged latency of nerve conduction" in a 22 year old male and suggested that the muscle weakness was more likely to be the result of a neurogenic rather than myopathic process. Procopis and Turner ${ }^{21}$ showed mild myopathic changes on EMG in one patient. Muscle biopsy and nerve conduction studies were normal. Muscle biopsy from a sib was said to show a pattern of neurogenic muscle atrophy. In the present study, creatine kinase was normal in all three patients tested. A muscle biopsy in case 1 was normal although the EMG findings were interpreted as possibly myopathic in origin. We feel that the clinical features and ultrasound findings certainly seem to indicate neuromuscular disease but supplementary investigations have failed to determine the nature of the underlying abnormality.
In summary, we have observed an unusual cataplexy-like phenomenon in three subjects with the Coffin-Lowry syndrome and we also describe muscle ultrasound changes suggestive of neuromuscular dysfunction in four cases. Recently, mutations in the Rsk- $2^{4}$ gene have been reported in subjects with Coffin-Lowry syndrome. Rsk-2 is a member of a family of growth factor regulated serine-threonine kinases, but at present the known functions of Rsk-2 provide no clues as to the nature of these phenomena and we are uncertain as to the underlying mechanisms.

We would like to thank Mrs Jean Hyslop of the Department of Medical Illustration for preparation of the clinical photographs.

1 Coffin GS, Siris E, Wegienka LC. Mental retardation with osteocartilaginous

2 Lowry B, Miller JR, Fraser FC. A new dominant gene mental retardation syndrome: association with small stature, tapering fingers, characteristic facies, and possible hydrotapering fingers, characteristic facies, and poss
cephalus. Am $\mathcal{F}$ Dis Child 1971;121:496-500.

3 Young ID. The Coffin-Lowry syndrome. $\mathcal{F}$ Med Genet 1988; 25:344-8.

4 Trivier E, De Cesare D, Jacquot S, et al. Mutations in the kinase Rsk-2 associated with Coffin-Lowry syndrome. Nature 1996;384:567-70.

5 Heckmatt JZ, Leeman S, Dubowitz V. Ultrasound imaging in the diagnosis of muscle disease. $\mathcal{F}$ Pediatr 1982;101:65660.

6 Ishida Y, Oki T, Ono Y, Nogami H. Coffin-Lowry syndrome associated with calcium pyrophosphate crystal deposition in the ligamenta flava. Clin Orthop Rel Res 1992;275:144in the

7 Fryns JP, Vinken L, van den Berghe H. The Coffin syndrome. Hum Genet 1977;36:271-6.

8 Partington MW, Mulley JC, Sutherland GR, Thode A, Turner G. A family with the Coffin-Lowry syndrome revisited: localisation of CLS to Xp21-pter. $\mathrm{Am} \mathcal{F} \mathrm{Med}$ Genet 1988;30:509-21.

9 Manford MRA, Fish DR, Shorvon SD. Startle provoked epileptic seizures - features in 19 patients. $f$ Neurol Neurosurg Psychiatry 1996;61:151-6.

10 Brown P, Rothwell JC, Thompson PD, Britton TC, Day BL, Marsden CD. The hyperekplexias and their relationship to the normal startle reflex. Brain 1991;114:1903-28.

11 Adams RD, Victor M, Ropper AH, eds. Principles of neurology. New York: McGraw-Hill, 1997:101-2.

12 Yoss RE, Daly DD. Criteria for the diagnosis of the narcoleptic syndrome. Staff Meet Mayo Clin 1957;32:320 8.

13 Kandt RS, Emerson RG, Singer HS, Valle DL, Moser HW. Cataplexy in variant forms of Niemann-Pick disease. Ann Neurol 1982;12:284-8.

14 Stephenson JBP. Fits and faints. London: Mackeith Press, 1990:144.

15 Vossler DG, Wyler AR, Wilkus RJ, Gardner-Walker G, Vlcek BW. Cataplexy and monoamine oxidase deficiency in Norrie disease. Neurology 1996;46:1258-61.

16 Matsuki K, Grumet FC, Lin X, et al. DQ (rather than DR) Matsuki K, Grumet FC, Lin X, et al. DQ (rather than DR)
gene marks susceptibility to narcolepsy. Lancet 1992;339: gene

17 Guilleminault C, Gelb M. Clinical aspects and features of cataplexy. Adv Neurol 1995;67:65-77.

18 Heckmatt JZ, Dubowitz V, Leeman S. Detection of pathological change in dystrophic muscle with B-scan ultrasound imaging. Lancet 1980:i;1389-90.

19 Hunter AGW, Partington MW, Evans JA. The Coffin-Lowry syndrome. Experience from four centres. Clin Genet 1982 21:321-35.

20 Vine DT, Gold JT, Grant AD. Etiology of the weakness in Coffin-Lowry syndrome. Am $\mathcal{F}$ Hum Genet 1986;39:A85.

21 Procopis PG, Turner B. Mental retardation, abnormal fingers, and skeletal anomalies: Coffin's syndrome. $\mathrm{Am} \mathcal{F}$ Dis Child 1972;124:258-61. 